\title{
Infrared Spectroscopy of the Centaur Asbolus with the ESO-Very Large Telescope
}

\author{
Catherine de Bergh, Maria-Antonietta Barucci, Aurélie Le Bras, \\ Jennifer Romon \\ Observatoire de Paris, 92195 Meudon, France
}

\author{
Bernard Schmitt, and Jean-Gabriel Cuby \\ Lab. Planétologie Grenoble, France, and ESO-Santiago, Chile
}

\begin{abstract}
As part of an observing programme with the ISAAC infrared spectrometer at the 8-m Antu telescope of the ESO-Very Large Telescope (Chile) devoted to the study of the surface composition of Kuiper Belt Objects and Centaurs, we obtained, in May 1999, spectra of the red Centaur Asbolus in the $\mathrm{J}, \mathrm{H}$ and $\mathrm{K}$ bands. The spectra appear featureless.
\end{abstract}

The Centaurs are minor planets with orbits having semi-major axes between those of Jupiter and Neptune. Centaurs are probably Kuiper Belt objects (KBOs) whose orbits have been modified by perturbations by the giant planets. There is a wide dispersion in the colors of the Centaurs, very similar to what is found for TNOs. Their colors range from neutral for 2060 Chiron (which has shown signs of cometary activity) to very red for 5145 Pholus and 7066 Nessus. Information on their surface composition has been obtained for only very few objects. In particular, when we planned our Asbolus observations, near-infrared spectroscopy (a spectral range particularly important to look for diagnostic features of plausible surface materials) had been carried out for only three Centaurs: 2060 Chiron, 5145 Pholus and $1997 \mathrm{CU}_{26}$.

A composite spectrum of Pholus that includes all published spectroscopic data from 0.45 to $2.5 \mu \mathrm{m}$ has been modelled by a mixture of olivine, a complex organic compound called Titan "tholin", ices of $\mathrm{H}_{2} \mathrm{O}$ and $\mathrm{CH}_{3} \mathrm{OH}$, and carbon on its surface (Cruikshank et al. 1998). Water ice has been found on Chiron and on $1997 \mathrm{CU}_{26}$ (see e.g. Luu et al. 2000, and Brown and Koresko 1998).

In May 1999, we carried out at ESO-VLT observations of 8405 Asbolus (estimated diameter: $74 \mathrm{~km}$; heliocentric distance: $8.94 \mathrm{AU}$ ) with the ISAAC spectrometer in its low resolution mode. Using the color indexes measured by Davies et al. (1998), the estimated J, $\mathrm{H}$ and $\mathrm{K}$ magnitudes at the time of our observations are, respectively, 16.8, 16.5 and 16.4. The spectra of Asbolus in each spectral range ( $\mathrm{J}, \mathrm{H}$ and $\mathrm{K}$ ) were divided by calibrator (solar-type star or C-type asteroids) spectra corresponding to the best match in air-mass. To increase the signal-to-noise ratio of our spectra we degraded the resolution by a factor of 5 . We see no obvious feature in the resulting spectra (resolution: 100), in particular no feature that could be attributed unambiguously to water ice.

To help us investigating the composition of the surface of Asbolus, we attempted to fit a composite spectrum that included visible spectra recorded at the 
ESO-3.6 $\mathrm{m}$ telescope in 1998 by Barucci et al. (1999) and our VLT near-infrared spectra with modelled spectra.

In our first category of models, we considered a simple geographical distribution of Titan tholins (included in models of 5145 Pholus to account for the steep slope of the visible) and amorphous carbon (a dark spectrally featureless material) to account for the low albedo and the slight infrared slope. In our second category of models, we replaced the tholins by a kerogen-type organic compound. Kerogens are complex organic material produced on the Earth primarily by geologic processing of biologic materials. Kerogen-like compounds, which are found in carbonaceous meteorites, could be constituents of the dark reddish surface of D-type asteroids (see e.g. Gradie \& Veverka 1980). Although few spectra of kerogens exist in the literature, the published spectra that correspond to different types of kerogens have slightly different spectral characteristics. We selected a type of kerogens studied in the laboratory by Clark (see Figure 8 from Cruikshank 1987) that has a spectral slope in the visible very similar to that of Titan tholins and no absorption in the infrared. These kerogens can therefore replace the Titan tholins in our models. We still need to include a very dark compound (here we choose amorphous carbon), but, due to the much smaller albedo of the kerogens, less of it is needed.

While the general shape of the spectrum could be reproduced by a mixture of $50 \%$ kerogen and $50 \%$ amorphous carbon, the best fit was obtained with most of the surface covered by sub-micron amorphous carbon grains and the remaining by tholins (see Barucci et al. 2000). When we added water ice, we found that, in the case of our tholins plus amorphous carbon areal mixture, no more than a few $\%$ of the surface (depending on the grain size) can be covered with pure water ice in order to keep the strong $2-\mu \mathrm{m}$ ice band within the noise level in the $\mathrm{K}$ region. In the case of the kerogen plus amorphous carbon mixture, no good fit could be obtained when water ice was included. More details can be found in Barucci et al. (2000).

Different types of surface representations need to be investigated. It is also essential to look at other parts of the Centaur since its surface may be heterogeneous, as seems to be the case for the Kuiper Belt Object 1996 TO $_{66}$ (Brown et al. 1999).

\section{References}

Barucci, M.A., Lazzarin, M., Tozzi, G.P. 1999, AJ, 117, 1929

Barucci, M.A. et al. 2000, A\&A, 357, L53

Brown, M.E. \& Koresko C. 1998, ApJ, 505, L65

Brown, R.H. et al., 1999, ApJ, 519, L101

Cruikshank, D.P. 1987, Adv. Space Res., 7, 109

Cruikshank, D.P. et al. 1998, Icarus, 135, 389

Davies, J.K. et al. 1998, Icarus, 134, 213, 1998

Gradie, J. \& Veverka J., 1980, Nature, 283, 840

Luu, J.X., Jewitt, D.C., Trujillo, C. 2000, ApJ, 531, L151 